Document downloaded from:

http://hdl.handle.net/10251/60240

This paper must be cited as:

Blanes Campos, C.; Ortiz Sánchez, MC.; Mellado Arteche, M.; Beltrán Beltrán, P. (2015). Assessment of eggplant firmness with accelerometers on a pneumatic robot gripper. Computers and Electronics in Agriculture. 113:44-50. doi:10.1016/j.compag.2015.01.013.

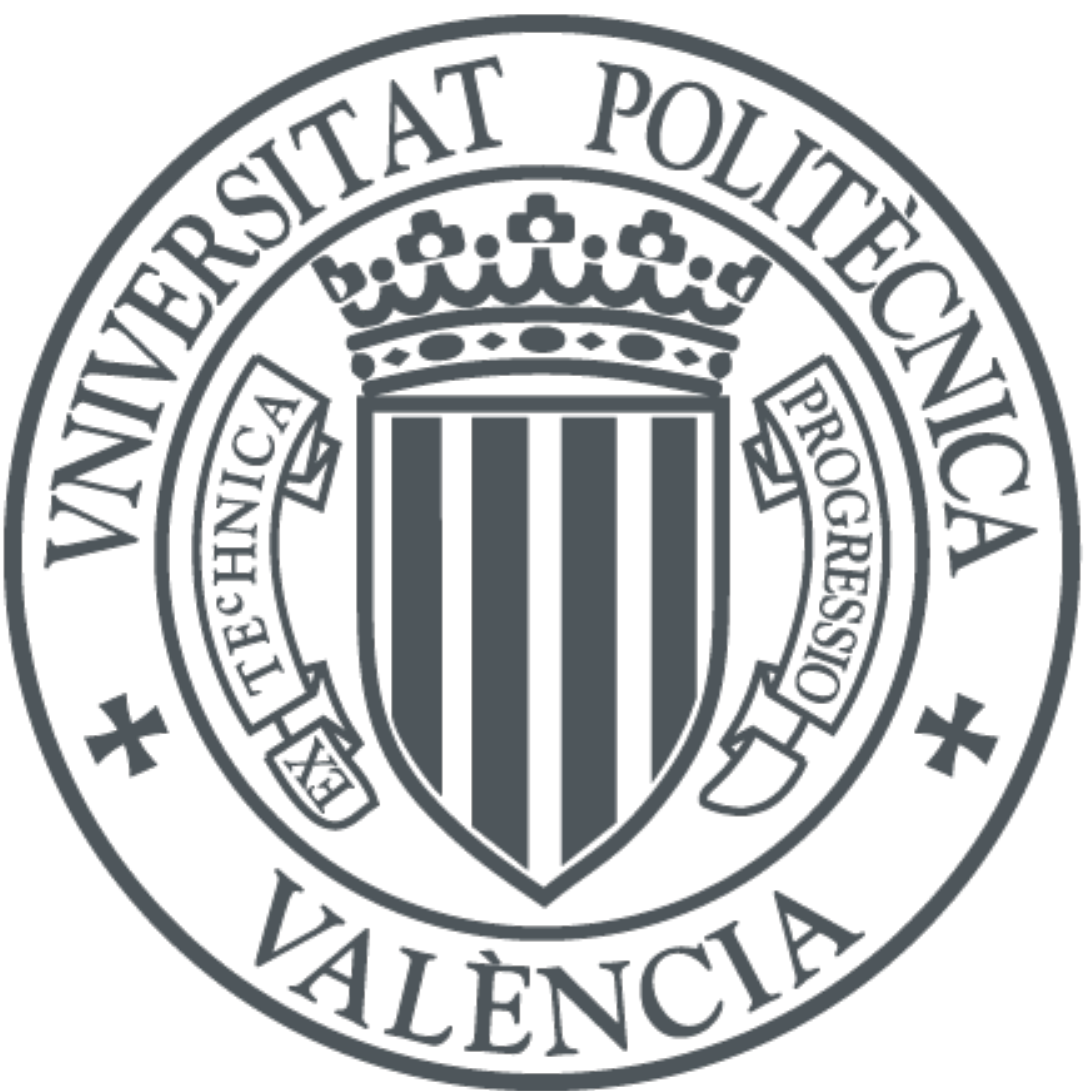

The final publication is available at

http://dx.doi.org/10.1016/j.compag.2015.01.013

Copyright Elsevier

Additional Information 


\title{
Assessment of eggplant firmness with accelerometers on a pneumatic robot gripper
}

\author{
Carlos Blanes $^{1, *}$, Coral Ortiz ${ }^{2}$, Martin Mellado ${ }^{1}$ and Pablo Beltrán ${ }^{1}$ \\ 1 Instituto de Automática e Informática Industrial / Universitat Politècnica de València \\ 2 Departamento de Ingeniería Rural y Agroalimentaria / Universitat Politècnica de València \\ Edificio 8G. Acceso D. $3^{\text {a }}$ Planta. Camino de Vera s/n, 46022 Valencia (Spain) \\ E-Mails: carblac1@ai2.upv.es; cortiz@dmta.upv.es; martin@ai2.upv.es; pabbelbe@ai2.upv.es
}

* Author to whom correspondence should be addressed;

Tel.: +34963877007 ext: 88233; Fax: +34963879816

\begin{abstract}
A pneumatic robot gripper capable of sorting eggplants according to their firmness has been developed and tested. The gripper has three fingers and one suction cup. Each finger has an inertial sensor attached to it. One of the fingers adapts to and copies the shapes of eggplants when the jamming of its internal granular material changes from soft to hard. The other fingers adapt to the shape of the eggplant with the use of extra degrees of freedom. Specific software acquires and processes the information obtained with the inertial sensors and generates 16 independent variables extracted from the signals. A total of 234 eggplants were selected and tested on the same day with the robot gripper, during the pick-and-place operation, and with a destructive firmness tester. The non-destructive parameters extracted from the gripper finger accelerometers were used to build and validate a partial least square model, with a calibration regression coefficient of $r=0.87$ and a high prediction performance $(r=0.90)$. Furthermore, from the results of the paper, it has been seen that the procedure can be simplified by using only two non-destructive impacts and one uniaxial accelerometer to assess eggplant firmness. The non-destructive assessment of firmness while grasping agricultural products in pick-and-place operations could be implemented in many prehensile pneumatic robot grippers. This technique could mean an important advance in the hygienic postharvest handling of fruits and vegetables.
\end{abstract}

Keywords: Tactile sensing; grasp contact; Pick \& Place operations; postharvest handling; non-destructive measurements; quality assessment 


\section{Introduction}

Increasing consumer awareness of the quality of fresh fruit and vegetables highlights the urgent need for research activities in order to assess the main quality parameters (Butz et al., 2005). Nowadays, sorting machines, based on vision analysis, are being used to grade fruits by size, color, stem location, and external blemishes in fruit and vegetable packing lines (Blasco et al., 2003). Many manufacturers have developed commercial online grading systems based on color and NIR sensors to classify fruits according to their internal chemical quality, especially sugar content (Nicolaï et al., 2007). Unfortunately, no scientific evidence is available regarding the accuracy of these systems. Texture is one of the key quality attributes of fruit and vegetables, and it is used to monitor and control quality along the food value chain (Chen and Opara, 2013).

Some commercial devices have been developed to assess fruit and vegetable firmness during sorting operations, such as the Sinclair Internal Quality-Firmness Tester (www.sinclair-intl.com), the Greefa Intelligent Firmness Detector (www.greefa.com) and the Aweta Acoustical Firmness Sensor (www.aweta.com). However, the results of these online non-destructive measurements are still far below those obtained with traditional laboratory destructive firmness measurements (Kupferman, 2007).

The human hand can handle and feel horticulture products at high speed. On most packaging lines, certain quality attributes related to texture are sorted by operators during manual handling despite the risk of contamination and the spreading of foodborne diseases (Todd et al., 2010). Robots can be easy-to-clean and can ensure a constant quality, speed and reliability (Petterson et al., 2010), while also clearly being suitable for automating primary and secondary packaging operations (Wilson, 2010; Kondo, 2010). The lack of robot grippers able to adapt to the product and the inability of sensors to sort products like operators makes it difficult to introduce robots into the horticultural packaging sector. Robot grippers for handling horticulture products can be designed on the basis of different technologies (Blanes et al., 2011). Multifingered robot hands are the most promising approach to human dexterity but most of them are both too fragile and complex, as well as too expensive, have a low operating speed, and are not robust enough for industrial applications, where far less complex solutions do accomplish the requirements. A gripper with underactuated mechanisms increases the degrees of freedom of the fingers and consequently their adaptability without increasing the number of actuators, thereby reducing complexity. This solution is well-suited for industrial applications (Meijneke et al., 2011) and is starting to be used in the horticulture industry (Lacquey, www.lacquey.nl). The jamming of granular materials 
allows adaptation to products with irregular shapes. It copies the product shape and ensures a wide contact surface and, therefore, reduces the maximum pressure applied (Brown et al., 2010).

Tactile sensors in robot grippers add the ability to feel, or sense, the product during handling processes. They can be classified according to sensor location (Dahiya et al., 2010) as intrinsic sensors, when the sensor is not in direct contact with the product, and extrinsic sensors, when the sensor needs to be in contact with the product (Tegin, 2005). Tactile sensors can also be classified into two main groups according to the property measured: static or passive and dynamic or active. Passive sensing analyzes the physical properties of static tactile contacts whereas in active sensing, motion is used dynamically to extract more information. Intrinsic sensors do not run the risk of being damaged by wear and tear during handling, and therefore have advantages when it comes to grading fruits and vegetables. They can be embedded during manufacturing processes (Jentoft et al., 2014; Issa et al., 2013) and they are able to evaluate product softness in continuum robots (Xu and Simaan, 2008). Product hardness has been evaluated with a quasi-static intrinsic sensor which measures the sensor displacement, while the internal sensor chamber is deformed under pneumatic (Shikida et al., 2003) or magnetic actuation (Hasegawa et al., 2006). Accelerometers can be used as active intrinsic sensors for texture perception (Yoshioka et al., 2007). These sensors analyze the response of a tri-axial accelerometer fitted to a probe that slides over several surfaces. Accelerometers on a pneumatic gripper can monitor the instant in which the contact occurs and when combined with a force sensor it becomes possible to regulate the grasping force (Chiara Lanni, 2008). Some developments have been made in the grading of fruits or vegetables with robot grippers with intrinsic active sensors by means of an online algorithm that analyzes the current of an electric motor gripper (Naghdy, 1996) and extrinsic sensor with a piezoresistive flexible tactile sensor (Bandyopadhyaya et al., 2014). Extrinsic tactile sensors can evaluate product hardness by using a piezoelectric transducer (Kimoto and Matsue, 2011) or in combination with a pressure sensor (Omata et al., 2004).

The objective of this research is to develop and test a pneumatic robot gripper capable of manipulating eggplants and assessing their firmness using accelerometers attached to the fingers as intrinsic tactile sensors.

\section{Materials and methods}

Two different approaches were used to evaluate firmness, a non-destructive approach and a destructive one, both carried out on the same day with 234 eggplants. In the non-destructive approach, each eggplant was grasped with a pneumatic gripper equipped with inertial sensors attached to its fingers. In the destructive approach, two 
destructive tests were carried out on each eggplant to evaluate firmness by measuring certain destructive mechanical parameters. The eggplants were acquired from eight different traders at the central market in Valencia (a city close to an important eggplant production area in Spain) in order to cover a wide range of possible variations in the ripening stage (from $48 \mathrm{~h}$ to 5 days after harvesting date). They were selected 24 hours before the day of the experiment, stored under refrigerated conditions $\left(5^{\circ} \mathrm{C}, 65 \%\right.$ relative humidity $)$, and allowed to warm up at room temperature for 2 hours before the tests.

\subsection{Non-destructive test}

A gripper was specifically designed for handling objects with the cylindrical and conical shapes typical of eggplants.

Previous results showed the possibility of classifying eggplants into firmness categories according to the parameters obtained during handling by the robot gripper. In a preliminary test, 30 eggplants were pre-classified into three groups according to their destructive firmness. A discriminant analysis was carried out to classify the vegetables in the firmness categories according to the information recorded by the accelerometers attached to the gripper, the result being a $90 \%$ rate of correctly classified fruits (Blanes et al., 2013).

The gripper is able to adapt to irregular shapes because its finger motion is underactuated, the number of actuators is lower than the degrees of freedom of the fingers, and the finger A has a granular material that adapts to and copies the shape of the eggplant (Figure 1). 


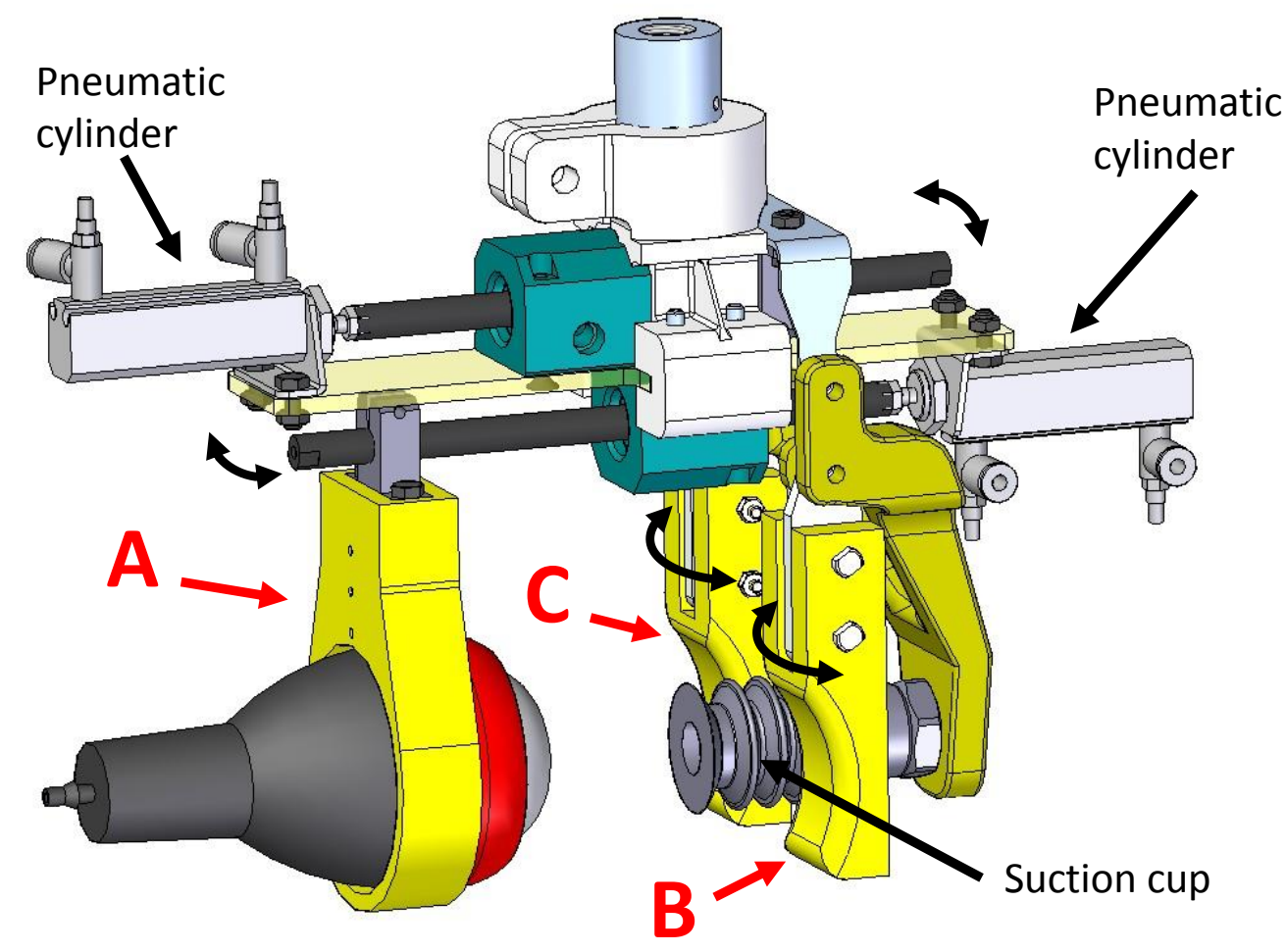

Figure 1. Robot gripper model with finger types and degrees of freedom.

The gripper offers a parallel movement actuated by two pneumatic cylinders. Rod cylinders can rotate freely. The gripper has three fingers (A, B \& C) and one suction cup located between fingers B and C. Finger A touches the product from one side while the other two secure it from the opposite side. The hardness of finger $\mathrm{A}$ is controlled by the jamming process of its internal granular material, in this case coriander seeds (mean values are: diameter $0.003 \mathrm{~m}$, density $783 \mathrm{Kg} / \mathrm{m}^{3}$, rupture force $8.1 \mathrm{~N}$, static coefficient of friction against galvanized iron 0.44). Its area of contact is a latex membrane that adapts gently to the shape of any eggplant, the membrane being kept rigid by applying suction inside the membrane. Fingers B and C can also rotate freely around a vertical axis.

\subsubsection{Robot operation}

The gripper is attached to an ABB IRB 340 FlexPicker robot. A robot program controls both the movements of the robot and the operation of the gripper. A vacuum generator with a blow function controls the hardness of finger A, another vacuum generator controls the suction cup, and an electro-valve controls the opening/closing of the fingers of the gripper. 
The robot gripper methodology was developed as a consequence of previous experiments. All the eggplants are placed manually, one by one, on the conveyor belt in a similar location and orientation. The robot program is started manually and all further steps are controlled by the robot. The robot moves the gripper down until reaching a predefined position where the eggplant is located between the fingers (Figure 2). Before grasping each eggplant, a positive pressure is exerted for 0.03 seconds to inflate the latex membrane and ensure smooth behavior of the granular material before contact. The latex membrane is then deflated smoothly. During this deflating period the granular material becomes loose and soft and the gripper fingers start to close. The latex membrane of finger A comes gently into contact against the eggplant and adapts to its shape. Fingers B and C then rotate freely around their vertical axes and around the pneumatic piston rod while in contact with the eggplant, the accelerometers being kept perpendicular to the surface of the eggplant. After 0.17 seconds a negative pressure is applied inside the membrane of finger A and its surface goes from being soft to a rigid state. The robot moves the gripper upwards. The gripper fingers open and close five times to produce five small shocks against the eggplant. During this period, the suction cup remains activated and the eggplants are held and in contact with fingers $\mathrm{B}$ and $\mathrm{C}$. The robot sends the signal to open the gripper for 0.06 seconds, and after that another signal to close it for 0.96 seconds. With the current experimental process 9.5 seconds are needed to classify each eggplant (Table 1).

\begin{tabular}{lcc} 
Robot Operation & Current (s) & Optimized (s) \\
\hline \hline Move down & 0.93 & 0.2 \\
Blow up & 0.03 & 0.03 \\
Close gripper & 0.17 & 0.1 \\
Stabilization & 0.13 & 0.133 \\
Wait & 1 & \\
Move up & 0.7 & 0.2 \\
Stabilization & & 0.133 \\
Wait (begin loop) & 0.4 & \\
\hline Open gripper & 0.06 & 0.05 \\
Close gripper & 0.06 & 0.05 \\
Stabilization & 0.133 & 0.133 \\
Wait & 0.767 & \\
(end of loop) & $(5$ times) & $(2$ times) \\
\hline Motion to place & & 0.3 \\
Move down & 0.7 & 0.2 \\
Wait & 0.2 & 0.1 \\
Open gripper & 0.13 & 0.1 \\
\hline \hline Total time & $\mathbf{9 . 5 3}$ & $\mathbf{1 . 9 6}$
\end{tabular}

Table 1. Current robot process for assessing the firmness of each eggplant and suggested optimized process. 
On a robotic industrial packaging line the software, such as ABB PickMaster, identifies the eggplants, together with their location and orientation, by means of a camera situated above the conveyor belt, which has an encoder connected to the software that coordinates the movements of the robot. The place where the eggplants are released can be modified based on the firmness estimated by this gripper.

\subsubsection{Tactile sensor and control software}

Each gripper finger has, attached to the external side, one biaxial analog accelerometer ADXL278 that works in the range of $+/-50 \mathrm{~g}$. The accelerometers are intrinsic tactile sensors, which are not in direct contact with the eggplants, with the capability of registering $\mathrm{x}$ and $\mathrm{y}$ accelerations for each finger (Figure 2).

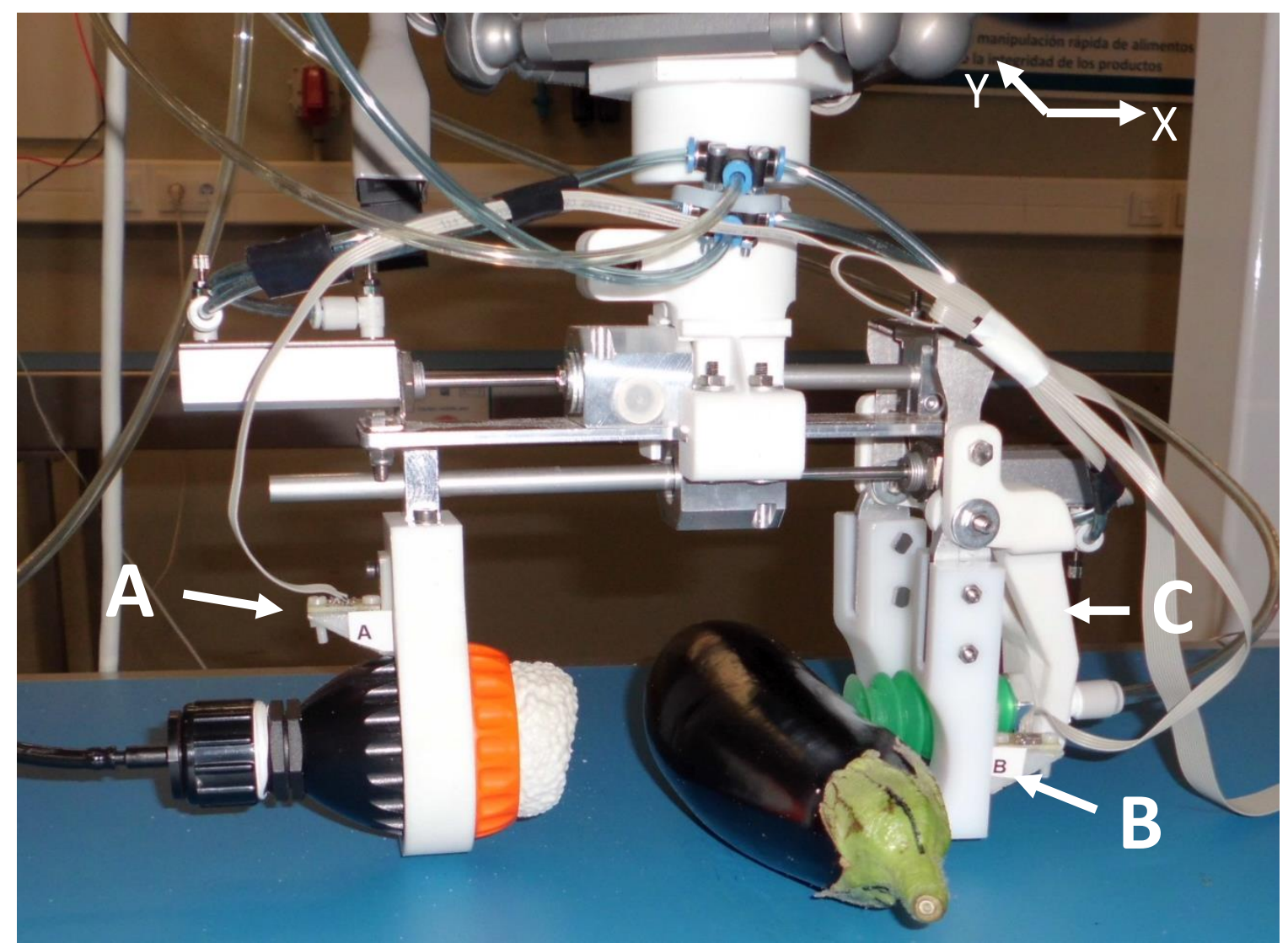

Figure 2. Robot gripper with inertial sensors.

Six acceleration signals, Ax (accelerometer A axis $\mathrm{x}$ ), Ay, Bx, By, Cx and $\mathrm{Cy}$ were collected and sent to a computer by means of an NI USB-6210 A/D data acquisition module. Signals were sampled at $30 \mathrm{KHz}$ and lowpass filtered at $1500 \mathrm{~Hz}$. As in preliminary studies (Blanes et al., 2013), no clear correlation was found between eggplant hardness and acceleration measured along the $y$ axis.

In this research, new software was programmed in LabVIEW for signal processing, this application allowing the computation of 24 independent parameters belonging to three different groups: 
- Parameters extracted from the analysis of the signal while contact occurs

These are extracted from the period of time, $t_{0}$ to $t_{1}$ (Figure $3 a$ ), in which the gripper fingers come into contact with the eggplant. Equations 1 to 3 determine four independent parameters VAx, VBx, VCx, Vtxm and one dependent one, $\mathrm{Vt}$, where $\mathrm{m}$ is the mass of the eggplant.

$$
V A x=\int_{t_{0}}^{t_{1}} A x^{2} d t ; V B x=\int_{t_{0}}^{t_{1}} B x^{2} d t ; V C x=\int_{t_{0}}^{t_{1}} C x^{2} d t
$$

$V t=V A x+V B x+V C x ;(2)$

$V t x m=m * V t$

- Maximum decelerations

Maximum deceleration is the highest value achieved in each signal and determines three independent parameters $\operatorname{Max} A x=A x\left(t_{M A x}\right), \operatorname{MaxBx}=B x\left(t_{M B x}\right)$, and $\operatorname{MaxCx}=C x\left(t_{M C x}\right)$, where $\operatorname{Max} A x$ is the maximum value achieved in accelerometer $\mathrm{A}$ for direction $\mathrm{x}$, and $\mathrm{t}_{\mathrm{MAx}}$ is the time when the maximum acceleration is achieved in accelerometer $\mathrm{A}$ and direction $\mathrm{x}$.

- Deceleration severity

The deceleration severity is the slope of the line of the deceleration after the initial contact (Figure 3a) between the finger and the product. Rigid products suffer a more violent deceleration than soft products during a situation involving contact. During the gripper grasp action multiple impacts and rebounds occur. Therefore it is difficult to identify the exact time when the first contact between gripper finger and product occurs.

In order to estimate the deceleration severity for each signal, three methods have been developed:

- The first method (Figure $3 b$ ) calculates SlpAx as the slope of the line that passes from $A x\left(t_{\mathrm{MAx}}-1.3\right)$ to $\mathrm{Ax}\left(\mathrm{t}_{\mathrm{MAx}}-0.3\right)$, time being considered in milliseconds. Repeating this computation for $\mathrm{B}(\mathrm{x})$ and $\mathrm{C}(\mathrm{x})$, this method provides three independent parameters SlpAx, SlpBx, and SlpCx.

- The second one (Figure 3c) calculates MxSlpAx as the maximum value of the derivative functions of the signal $A x(t)$, from $A x\left(t_{\text {MAx }}-3\right)$ to $A x\left(t_{M A x}\right)$. This value is the maximum deceleration severity. $B y$ repeating this process for $\mathrm{B}(\mathrm{x})$ and $\mathrm{C}(\mathrm{x})$, three independent parameters MxSlpAx, MxSlpBx, and MxSlpCx are computed.

- The last method uses the derivative functions of the signals $\mathrm{Ax}(\mathrm{t}), \mathrm{Bx}(\mathrm{t})$, and $\mathrm{Cx}(\mathrm{t})$ (Figure 3c) and calculates the areas of these derivative functions in the intervals $\left[\mathrm{t}_{\mathrm{MAx}}-3, \mathrm{t}_{\mathrm{MAx}}\right],\left[\mathrm{t}_{\mathrm{MBx}}-3, \mathrm{t}_{\mathrm{MBx}}\right]$, and $\left[t_{\mathrm{MCx}}-3, \mathrm{t}_{\mathrm{MCx}}\right]$. This method computes three new independent parameters, MdSlpAx, MdSlpBx, and MdSlpCx. 


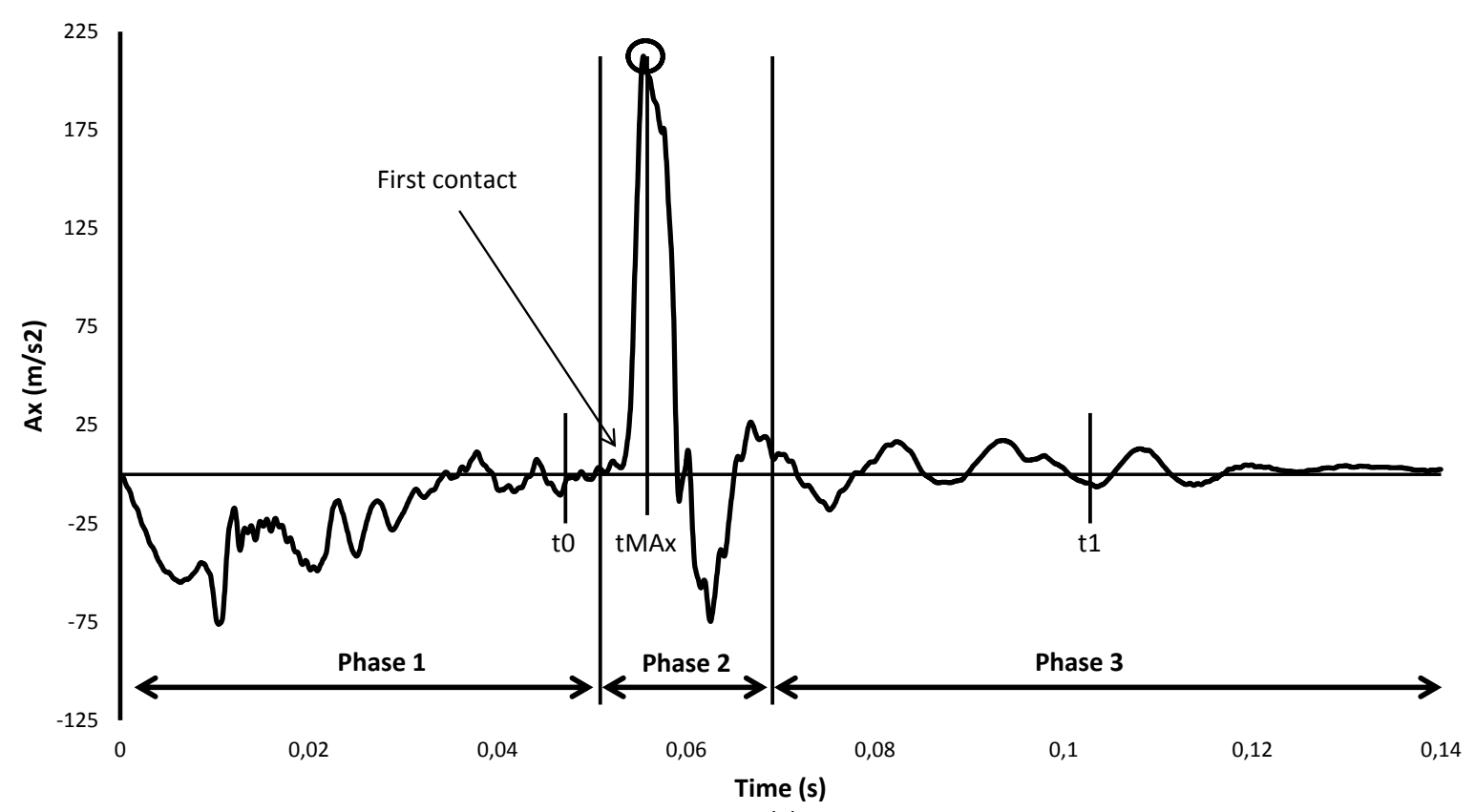

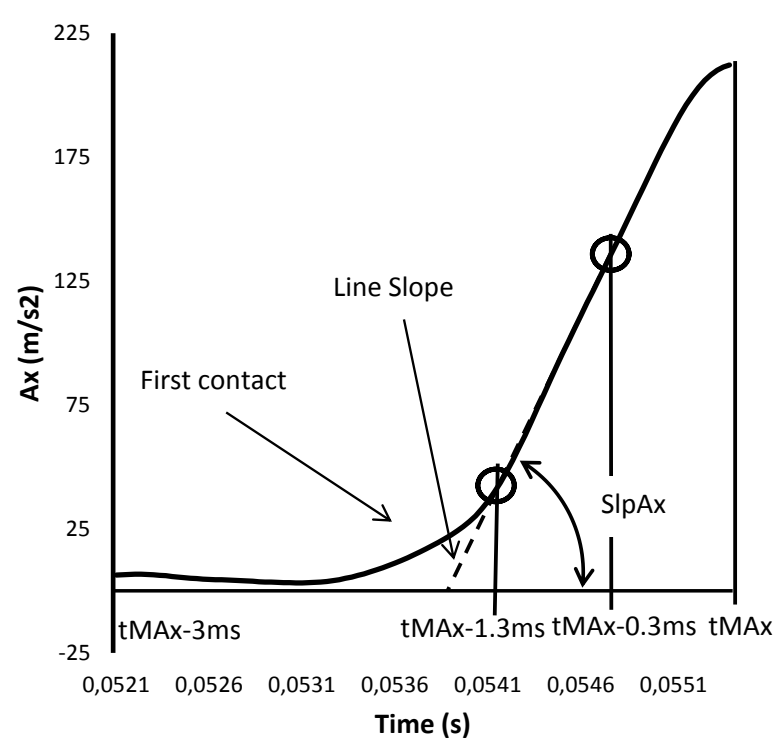

(b)

(a)

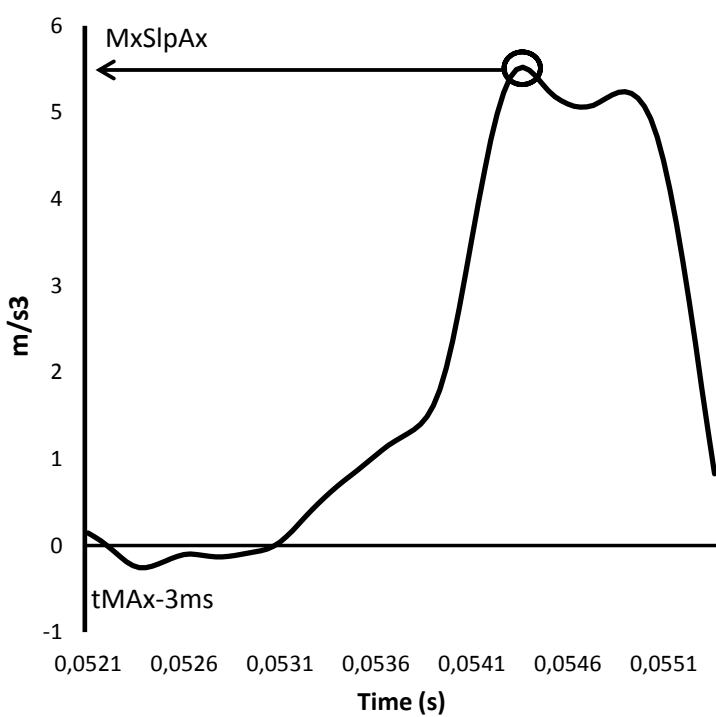

(c)

Figure 3. (a) An accelerometer signal from direction $x$, (b) the slope of the line, and (c) the maximum slope.

\subsection{Destructive tests for firmness evaluation}

In order to assess mechanical firmness through destructive techniques, and based on previous results, two tests were carried out: $5 \mathrm{~mm}$ compression of the whole eggplant with a spherical rod (10 $\mathrm{mm}$ diameter), and skin puncture with a cylindrical rod (1.5 $\mathrm{mm}$ diameter). A universal testing machine (Ibertest) with a digital dynamometer (Andilog Centor) that had a range of $0 \mathrm{~N}-500 \mathrm{~N}$, precision of $0.1 \%$, sampling frequency of $1.000 \mathrm{~Hz}$, and resolution of $0.025 \mathrm{~N}$ was used (Figure 4). 

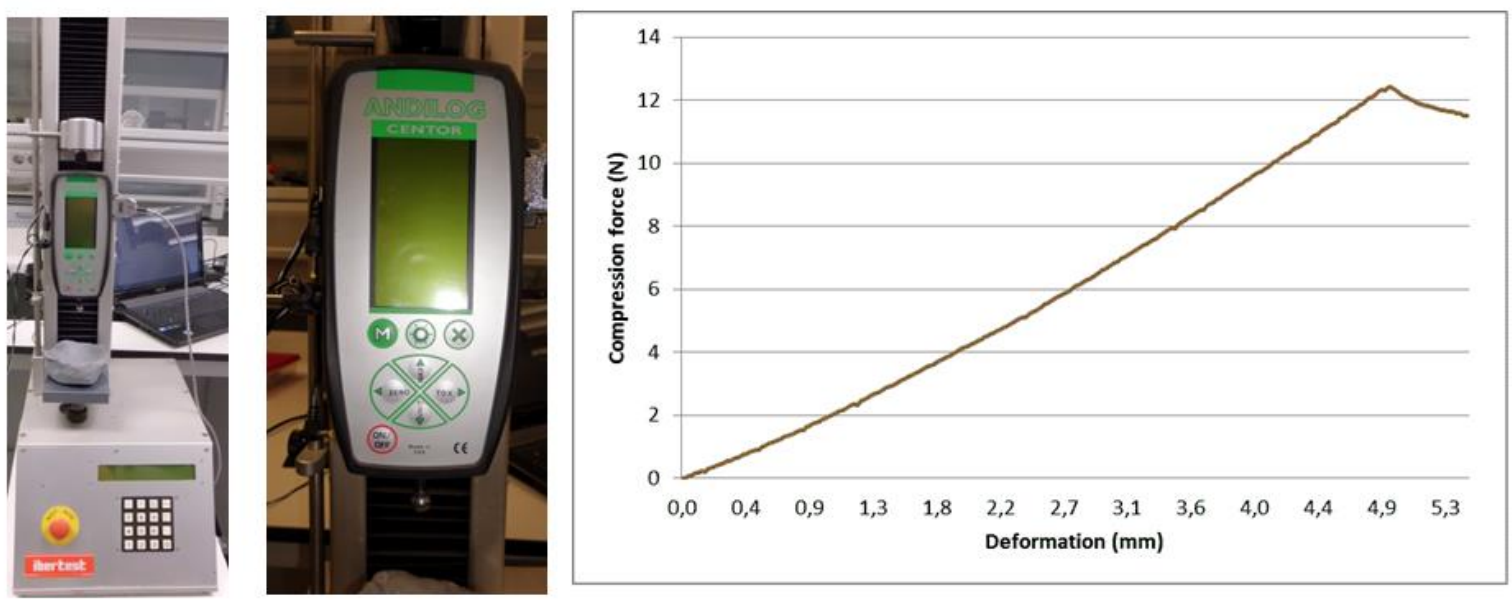

Figure 4. Universal testing machine for evaluation of eggplant firmness and the force deformation curve.

In both tests, the eggplants were placed on a piece of modeling clay. The tests were carried out at a probe speed of $1 \mathrm{~mm} / \mathrm{s}$. The maximum force required to make the puncture on the fruit surface $(\mathrm{N})$ was taken from the forcedeformation curve. The resistance to compression of the whole fruit was measured as the maximum compression force/deformation $(\mathrm{N} / \mathrm{mm})$ ratio taken from the compression force-deformation curve. Two repetitions were performed on each vegetable. The complementary parameters of weight, diameter and length were also measured.

\section{Results and discussion}

In spite of the wide variations in physical parameters (Table 2) the gripper was capable of grasping $100 \%$ of the eggplants without producing any visible damage. The gripper adapts to the irregular shapes thanks to the jamming system of finger $\mathrm{A}$, and to the underactuated movements of fingers $\mathrm{B}$ and $\mathrm{C}$.

$\begin{array}{lccccc} & \text { Weight }(\mathbf{g}) & \begin{array}{c}\text { Length } \\ (\mathbf{m m})\end{array} & \begin{array}{c}\text { Equatorial } \\ \text { diameter } \\ (\mathbf{m m})\end{array} & \begin{array}{c}\text { Maximum } \\ \text { puncture } \\ \text { force }(\mathbf{N})\end{array} & \begin{array}{c}\text { Compression } \\ \text { F/d (N/mm) }\end{array} \\ \text { Average } & 280.3 & 185.27 & 71.2 & 11.70 & 2.45 \\ \text { Minimum } & 160.7 & 132.0 & 59.8 & 8.49 & 0.87 \\ \text { Maximum } & 393.4 & 244.0 & 84.6 & 18.25 & 4.93 \\ \begin{array}{l}\text { Standard } \\ \text { deviation }\end{array} & 37.7 & 21.95 & 4.7 & 1.83 & 0.82\end{array}$

Table 2. Physico-mechanical parameters of eggplants, extracted from 234 eggplants. 
When products are grasped from the conveyor belt, the accelerometer signals have a wide variability and their firmness cannot be assessed. In this case, signals are affected by: the friction forces between the surfaces of the eggplant, conveyor belt, and gripper finger; the relative position and orientation between gripper and product; the dimensions of the eggplant; and by finger accommodation. These issues have been reduced by operating the robot gripper as detailed in section 2.1.1. During the open-close loop, the gripper fingers open for 0.06 seconds each time while the eggplant remains attached to fingers B and $\mathrm{C}$ due to the suction cup. As residual forces between fingers and eggplant are the same during the open-close loop, the gripper opens the same distance. Therefore, during the closing action, when the contact between the fingers and the eggplant starts, the energy of the gripper is always the same, and is independent of the size of the eggplant.

Since each gripper finger has an inertial sensor attached to it, the grasping action of each finger can be analyzed in detail in three different phases (Figure 3a). In the first phase, the finger starts its movement until it establishes contact. In the second one, the eggplant and the finger come into contact until the final approaching and pushing motion, where there is no relative movement between the product and the finger. In the third phase, the gripper and the dynamic forces find a static equilibrium with an under-damping movement while the product and the gripper move together. No relation was found between the physical parameters (weight, length, and diameter) and the destructive mechanical parameters, or with the non-destructive variables obtained from the tactile sensor signal. Furthermore, no relation was found between the maximum puncture force and the non-destructive variables obtained from the tactile sensor signal.

The variables obtained from the signal of the accelerometer on finger A showed higher repeatability compared to those of accelerometers $\mathrm{B}$ and $\mathrm{C}(\mathrm{VAx}$ repeatability $=91.7 \%$; $\mathrm{VBx}$ repeatability $=78.8 \%$; $\mathrm{VCx}$ repeatability $=$ 84.8\%). The best results were found with the parameters extracted from finger A. Therefore, the possibilities of firmness assessment increase as the adaptability of the gripper fingers to the product becomes higher.

A significant correlation was found between the destructive compression force deformation and non-destructive parameters obtained from the tactile sensor. The parameter with the strongest association with vegetable firmness measured as compression force deformation was VAx $(r=0.79, \mathrm{p}<0.001,99 \%)$. A correlation of 0.879 is considered fairly good bearing in mind that the destructive firmness (compression force deformation ratio) was not the actual firmness. 
To study the importance of the five impacts applied to the vegetable, a multiple regression test was carried out to relate compression force deformation to the parameters of the tactile sensor. The parameters obtained from the gripper sensors in the first impact explained less variability than the parameters obtained from the other impacts (adjusted $\mathrm{R}^{2}$ of 69.7 from the first impact, compared to 76.5, 75.3, 76.8 and 76.2 from the second, third, fourth, and fifth impacts, respectively, and 74.1 from the average of the five impacts, with $\mathrm{p}<0.0001,99 \%$ ). Applying only two impacts and processing the information of the second impact was enough to achieve the best estimation of firmness as results did not improve with more impacts. While the latex membrane adapted to the product, residual shearing forces remained between the product and the membrane. The gripper needed some extra force to overcome the residual forces to open during the first action of opening-closing, thus affecting the acceleration in the first impact of the loop and the results being worse than in the next open-close operations.

In order to build and validate a partial least square model (PLS) to predict firmness from the tactile sensor variables from the 234 eggplants that were tested, a sample of $78 \%$ of them were chosen at random. A PLS was performed to estimate the force deformation ratio in the compression test by using the non-destructive variables from the three tactile sensors in the second impact. The calibration model was also validated using one-leave-out cross-validation. A good regression coefficient was obtained during the calibration set with $r=0.87$, with the standard error of calibration (SEC) of $0.3510 \mathrm{~N} / \mathrm{mm}$ (Table 3). All the variables used in the PLS model denote a normal distribution according to their Standardized Kurtosis value.

\begin{tabular}{lcccccc}
\hline & N size & LV & \multicolumn{2}{c}{$\begin{array}{c}\text { Calibration } \\
\text { RMSEC }\end{array}$} & r & Cross validation \\
& & & RMSEP \\
\hline $\begin{array}{l}\text { Destructive } \\
\text { firmness }\end{array}$ & 12 & 4 & 0.87 & 0.17006 & 0.86 & 0.180048 \\
\hline
\end{tabular}

Table 3. Results of calibration and cross-validation of the PLS model.

When the model was used to predict the firmness of the remaining $22 \%$ of the eggplants, prediction results showed high prediction performance $(r=0.90)$, standard error of prediction (SEP) of $0.3153 \mathrm{~N} / \mathrm{mm}$ with a bias of $0.14007 \mathrm{~N} / \mathrm{mm}$ (Figure 5). 


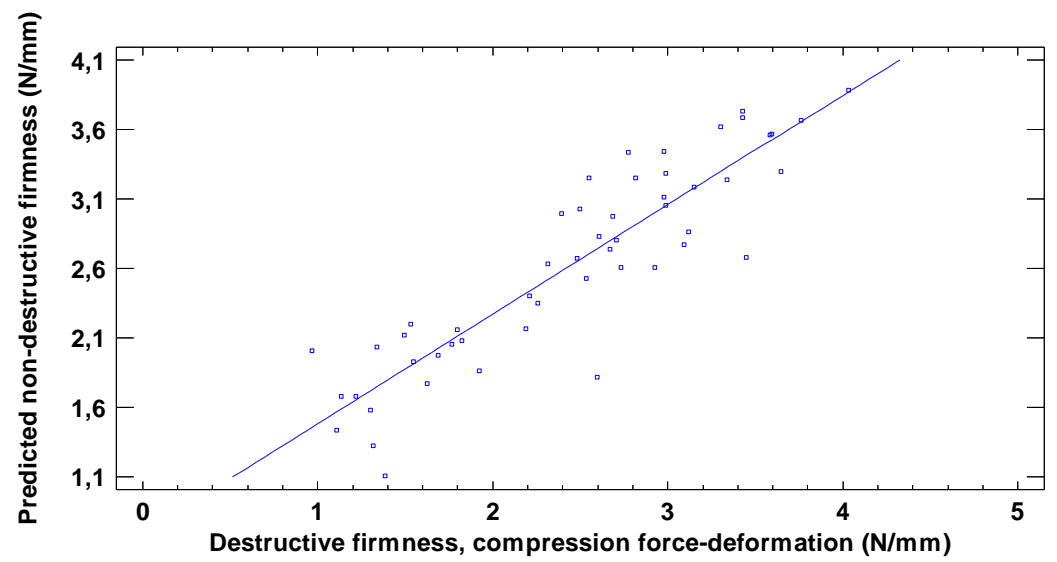

Figure 5. Predicted non-destructive firmness and observed values from the destructive test (compression force deformation $(\mathrm{N} / \mathrm{mm}))$.

Parameters obtained from acceleration in the direction of the y axis did not improve firmness estimation because the finger surfaces remain parallel during robot gripper impacts. The four extracted non-destructive parameters that were most related to destructive firmness were: VAx correlation coefficient 0.79 ; MaxAx correlation coefficient 0.78; MddcAx correlation coefficient 0.7091, and MxSlpAx 0.6156. Using only these four parameters extracted from finger A, a PLS model can be built and validated, with a calibration regression coefficient of $r=0.78$ and a high prediction performance $(r=0.89)$. Therefore, the complexity of the gripper could be reduced by using only one uniaxial accelerometer attached to finger A.

The non-destructive tactile sensor was capable of assessing the firmness of the whole eggplant (measured as compression force deformation) while grasping the vegetable. This result is in line with previous studies about non-destructive impact sensors used to assess fruit firmness that report that impact force and impact duration are directly related to fruit firmness (García-Ramos et al., 2005).

Robot operation can be simplified by reducing the number of impacts from five to two (Table 1). This process can be improved to achieve a process time of 1.96 seconds by increasing the pneumatic pressure to get a faster open/close gripper; increasing robot motion speed; adjusting the gripper range; with robot displacements from pick to place that take less than 0.3 seconds; and reducing the number of impacts. The same gripper without tactile sensing could save 0.469 seconds, 0.466 for two impacts, and 0.03 seconds on inflating finger A before grasping the eggplants. If the impacts for firmness assessment are produced during the robot movement between pick to place then the total process time is 1.66 seconds per eggplant. 


\section{Conclusions}

A pneumatic gripper with underactuated movements in each of its three fingers and a jamming system, capable of grasping elongated vegetables, has been designed. The robot gripper can be adapted to irregular eggplant shapes without producing any visible damage. Three inertial sensors attached to the three gripper fingers were able to non-destructively assess the firmness of the eggplant, the sensor information being processed while impact occurs with the vegetable during the pick-and-place operation. The non-destructive parameters extracted from the accelerometers on the gripper finger can be used to build and validate a partial least square model, with a calibration regression coefficient of $r=0.87$ and high prediction performance $(r=0.90)$. The parameters extracted from the analysis of the signal while impacts occur had a strong correlation with destructive firmness. This study has also shown that only two non-destructive impacts and one uniaxial accelerometer can be enough to assess the firmness of eggplants.

Pneumatic robot grippers like the one presented in this paper can be implemented in many industrial applications. This gripper can be implemented on an automatic robotic line without any special conveyors, robots or artificial vision systems. The software will coordinate the robot motion and decide on the place position based on the assessment of the firmness of the gripper. The installation of inertial sensors in robotic endeffectors is simpler than other similar tactile sensor solutions. With modifications to the shapes of the fingers, the gripper could be adapted for the hygienic postharvest handling and firmness assessment of other fresh fruits and vegetables.

\section{Acknowledgements}

This research is supported by MANI-DACSA project (ref. RTA2012-00062-C04-02), partially funded by the Spanish Government (Ministerio de Economía y Competitividad).

\section{References}

Bandyopadhyaya, I.; Babu, D.; Bhattacharjee, S.; Roychowdhury, J., 2014. Vegetable Grading Using Tactile Sensing and Machine Learning. In Advanced Computing, Networking and Informatics 1, 77-85

Blanes, C., Gomez, C., Ortiz, C., Mellado, M., 2013. Aubergine (Solanum melogena) firmness detection using a robot gripper during handling. VII Iberian AgroEngineering Conference, Madrid, August 2013. 
Blanes, C., Mellado, M., Ortiz, C., Valera, A., 2011. Technologies for robot grippers in pick and place operations for fresh fruits and vegetables, Span. J. of Agric. Res. 9, 1130-1141. (DOI 10.5424/sjar/20110904501-10)

Blasco, J., Aleixos, N., Moltó, E., 2003. Machine vision system for automatic quality grading of fruit, Biosystem Engineering 85(4), pp. 415-423. (DOI 10.1016/S1537-5110(03)00088-6)

Brown, E., Rodenberg, N., Amend, J., Mozeika, A., Steltz, E., Zakin, M.R., Lipson, H., Jaeger, H.M., 2010. From the Cover: Universal Robotic Gripper Based on the Jamming of Granular Material. Proc. Natl. Acad. Sci. U. S. A., 107(44), 18809-18814. (DOI 10.1073/pnas.1003250107)

Butz, P., Hofmann, C., Tauscher, B., 2005. Recent developments in noninvasive techniques for fresh fruit and vegetable internal quality Analysis. Journal of Food Science 70 (9), pp. 131-141.

Chen, L., Opara, U.L., 2013. Texture measurement approaches in fresh and processed foods. A review. Food Research International 51(2), pp. 823-835. (DOI 10.1016/j.foodres.2013.01.046)

Chiara Lanni, M.C. 2008. Regulation of Grasp Impact in Two-Finger Grippers, Proc. of the RAAD 2008, 17th Int. Workshop on Robotics 32.

Dahiya, R., Metta, G., Valle, M., Sandini, G., 2010. Tactile Sensing-From Humans to Humanoids, IEEE Trans. on robotics $26,1-20$.

García-Ramos, F.J., Valero, C., Homer, I., Ortiz-Cañavate, J. and Ruiz-Altisent, M., 2005. Non-destructive fruit firmness sensors: a review. Spanish Journal of Agricultural Research 3 (1), pp. 61-73.

Hasegawa, Y., Shikida, M., Sasaki, H., Itoigawa, K., Sato, K., 2006. An active tactile sensor for detecting mechanical characteristics of contacted objects, J. Micromech. Microeng. 16, 1625-1632.

Issa, Mirna Petkovic, Dalibor Pavlovic,Nenad Zentner, Lena., 2013. Sensor elements made of conductive silicone rubber for passively compliant gripper. Int. J. Advanced Manufact. Tech. 69, 1527-1536.

Jentoft, L. P., Dollar, A. M., Wagner, C. R. \& Howe, R. D., 2014. Intrinsic Embedded Sensors for Polymeric Mechatronics: Flexure and Force Sensing. Sensors 14, 3861-3870 
Kader, A.A., 2001. Quality assurance of harvested horticultural perishables. Acta Horticulturae553, Paper Read at 4th Intl. Conf. on Postharvest, pp. 51-56.

Kimoto, A., Matsue, Y., 2011 A New Multifunctional Tactile Sensor for Detection of Material Hardness, IEEE Trans. on Instrum. and Meas. 60, 1334-1339.

Kondo, N., 2010. Automation on fruit and vegetable grading system and food traceability, Trend Food Sci. Technol. 21(3), 145-152. (DOI 10.1016/j.tifs.2009.09.002)

Kupferman, E., 2007. Non-destructive firmness instrument testing: Apples and pears. Postharvest Information Network. Washington State University-Tree Fruit Research and Extension Center (accessed October 2014: (http://postharvest.tfrec.wsu.edu/EMK2007B.pdf)

Meijneke, C., Kragten, G., Wisse, M., 2011. Design and performance assessment of an underactuated hand for industrial applications. Mech. Sci. 2, 9-15. (DOI 10.5194/ms-2-9-2011)

Naghdy, F., Esmaili, M., 1996. Soft Fruit Grading using a Robotics Gripper, Int. J. of Robotics and Autom. 11, 93-101.

Nicolaï, B.N; Beullens, K; Bobelyn, E; Peirs, A; Saeys, W; Theron, K.I; Lammertyn, L. 2007. Nondestructive measurement of fruit and vegetable quality by means of NIR spectroscopy: A review. Postharvest Biology and Technology 46, 99-118

Omata, S., Murayama, Y., Constantinou, C.E., 2004. Real time robotic tactile sensor system for the determination of the physical properties of biomaterials, Sens. and actuators. A, Phys. 112, 278-285.

Petterson, A., Davis, S., Gray, J.O., Dodd, T.J., Ohlsson, T. 2010. Design of a magnetorheological robot gripper for handling of delicate food products with varying shapes. J. Food Eng. 98(3), 332-338

Shikida, M., Shimitzu, T., Sato, K., Itoigawa, K., 2003. Active tactile sensor for detecting contact force and hardness of an object, Sens. and actuators. A, Phys. 103, 213-218.

Tegin, J., 2005. Tactile sensing in intelligent robotic manipulation-a review, Ind. Robot 32 64-70. 
Todd, E.C.D., Michaels, B.S., Smith, D., Creig, J.D., Bartleson, C.A., 2010. Outbreaks where food workers have been implicated in the spread of foodborne disease. Part 9. Washing and drying of hands to reduce microbial contamination. J. Food Protection 10(19), 1937-1955.

Wilson, M., 2010. Developments in robot applications for food manufacturing, Ind. Robot 37, 498-502.

Xu, K., Simaan, N., 2008. An investigation of the intrinsic force sensing capabilities of continuum robots, IEEE Trans. on robotics $24,576-587$.

Yoshioka, T., Bensmaia, S.J., Craig, J.C., Hsiao, S.S., 2007. Texture perception through direct and indirect touch: an analysis of perceptual space for tactile textures in two modes of exploration, Somatosensory Mot. Res. 24, 53-70. 